\title{
APPLICATION OF MULTIVARIATE IMAGE ANALYSIS - QUANTITATIVE STRUCTURE TOXICITY RELATIONSHIP STUDY FOR MODELING THE TOXICITY OF PHENOL DERIVATIVES
}

\author{
ZAHRA GARKANI-NEJAD ${ }^{1 *}$ and FATHIYEH SALEHFARD ${ }^{2}$
}

\author{
${ }^{I}$ Chemistry department, Faculty of Science, Shahid Bahonar University of Kerman, Kerman, Iran \\ ${ }^{2}$ Chemistry department, Faculty of Science, Vali-e-Asr University, Rafsanjan, Iran
}

(Received: March 13, 2013 - Accepted: May 14, 2013)

\begin{abstract}
The toxicity of some phenol derivatives over tetrahymena pyriformis were modeled by using multivariate image analysis (MIA) descriptors applied to quantitative structure-toxicity relationship (QSTR) method. MIA descriptors are derived from pixels of two-dimensional (2D) chemical structures that may be built by using any appropriate drawing software. QSTR analysis has been done by using partial least square regression (PLS), correlation ranking-principal component regression (CR-PCR) and correlation ranking-principal component-artificial neural network (CR-PC-ANN) modeling methods. For the ANN models two different weight update functions of Levenberg-Marquardt (LM) and Scaled Conjugate Gradient (SCG) were investigated. Finally, obtained results using different linear and nonlinear modeling methods were compared, showing that PLS and CR-PC-ANN-SCG methods present high performance for prediction of the toxicity of phenol derivatives. Also, these results indicated that MIA descriptors may be useful to predict toxicity of phenol derivatives. Squared correlation coefficient $\left(\mathrm{R}^{2}\right)$ and standard error using the PLS method for the training set were 0.996 and 0.042 and for the test set were 0.996 and 0.048 , respectively. Squared correlation coefficient $\left(\mathrm{R}^{2}\right)$ and standard error using the CR-PC-ANN-SCG method were respectively, 0.999 and 0.002 for the training set, 0.999 and 0.001 for the test set and 0.999 and 0.002 for the validation set.
\end{abstract}

Keywords: Multivariate image analysis (MIA); Quantitative structure-toxicity relationship (QSTR); Principal component-artificial neural network (PCANN); phenol derivatives

\section{INTRODUCTION}

Phenols and their derivatives commonly exist in the environment. These compounds are used as the components of dyes, drugs and other organic substances. Phenol and its vapor are corrosive to the eyes and skin. Repeated or prolonged skin contact with phenol may cause dermatitis, or even second and third-degree burns ${ }^{1}$. The substance may cause harmful effects on the central nervous system and heart, resulting in dysrhythmia, seizures, and coma $^{2}$ Exposure may result in death and the effects may be delayed. Long-term or repeated exposure of the substance may have harmful effects on the liver and kidneys. There is no evidence to believe that phenol causes cancer in humans. The toxicity of phenol related to its hydrophobocity and also to the formation of phenoxyl radicals.

With the development of experimental chemistry, a great number of new compounds are synthesized every year. However, a large part of these compounds are not tested for fundamental or relevant thermodynamic and physicochemical properties or biological activities, which still remain unknown due to unavailability or no easily handling (toxic, odorous, etc.). A procedure able to predict the physicochemical properties and biological activities of untested compounds is required to evaluate these molecular features in fast and inexpensive way ${ }^{4}$. In recent years, numerous quantitative structure activity/property relationship (QSAR/QSPR) models have been introduced for calculating the physicochemical properties with various numerical descriptors of chemical structures. These relationships derive correlations between the similarities of individual compounds and their biological activities/chemical properties $^{5,6}$.

In QSAR studies, a regression model of the form $\mathbf{y}=\mathbf{X b}+\mathrm{e}$ may be used to describe a set of predictor variables $(\mathbf{X})$ with a predicted variable $(\mathbf{y})$ by means of a regression vector (b). However, the colinearity, which often existed between independent variables, creates a severe problem in certain types of mathematical treatment such as matrix inversion? ${ }^{7}$. A better predictive model can be obtained by ortogonalization of the variables by means of principal component analysis (PCA) and the consequent method is called principle component regression (PCR) ${ }^{8-10}$. In order to reduce the dimensionality of the independent variable space, a limited number of principal components (PCs) are used. Selection of significant and informative PCs is the main problem in almost all PCA-based calibration methods ${ }^{11-15}$. Different methods have been addressed to select the significant PCs for calibration purposes. One simple and common method, called correlation ranking, the factors are ranked by their correlation coefficient with the property to be correlated.

Artificial neural networks (ANN) are nonparametric nonlinear modeling techniques that have attracted increasing interest in recent years ${ }^{16-18}$. Nonlinear multivariate maps use a nonlinear transformation of the input variable space to project inputs onto the designated attribute values in the output space. The strength of modeling with layered, feed-forward artificial neural network lies in the flexibility of the distributed soft model defined by the weight of the network. Multilayer feed-forward neural networks trained with a back-propagation learning algorithm have become increasingly popular techniques ${ }^{19-21}$. The flexibility of ANN for discovering more complex relationships lead this method to find a wide application in QSAR/QSTR studies, as recently reviewed by Schneider and Wrede ${ }^{22}$.

A principal component-artificial neural network (PC-ANN) system, which combines the PCA with ANN, is another PCA-based calibration technique for nonlinear modeling between the PCs and dependent variables ${ }^{23,24}$. The problem of PC selection in PC-ANN is more serious than PCR because of unknown and complex relationship between PCs and dependent variables. A method for the selection of factors in PC-ANN is correlation ranking.

The present paper is focused on the application of 2D images, which are the proper structure of the compounds that can be drawn with aid of any appropriate program, as descriptors in QSTR. Multivariate image analysis (MIA) is known for a long time, but its application has mostly been extended to chemical problems by using spectral data, though it has other practical applications in the fields of scientific imaging. Geladi's and Esbensen's research ${ }^{25-28}$ provides illustrative examples of these. In the present study, the pixels of each image are correlated with the respective toxicities of 50 compounds, which are some phenol derivatives with toxicity over tetrahymena pyriformis. The results from this modeling are compared with those from a previous study ${ }^{29}$. The present method provides a very suitable analysis, since visual interpretation plays an important role in all forms of data analysis and is easily accessible to both newcomers and advanced users.

\section{THEORY}

\section{Artificial neural networks (ANNs)}

Artificial neural network (ANN) is a computer based system derived from the simplified concept of the brain in which a number of nodes, called processing elements or neurons, are interconnected in a netlike structure. The ANN characteristics have been found to be nonlinear, making them suitable for data processing in which the relationship between cause and results cannot be linearly defined. Three components constitute an ANN: the processing elements, the topology of connection between the nodes, and the learning rules. The structure of the network comprised of three node layers: an input, a hidden and an output layer, represented by i, h, and o, where they, respectively, indicate the number of nodes in the input layer, hidden layer, and output layer. The input nodes transferred the weighted input signals to the nodes in the hidden layer, and the same as the hidden nodes for the output layer. A connection between the nodes of different layers was represented by a weight 
$\mathrm{w}_{\mathrm{ij}}$, and during the training process, the correction of weight $\Delta \mathrm{w}_{\mathrm{ij}}$ was defined as the following:

$$
\Delta \mathrm{w}_{\mathrm{ij}, \mathrm{n}}=\mathrm{F}_{\mathrm{n}}+\alpha \Delta \mathrm{w}_{\mathrm{ij}, \mathrm{n}-1}
$$

Where $\Delta \mathrm{w}_{\mathrm{ij,n}, \mathrm{n}}$ is the change in the weight factor for each network node, $\alpha$ is the momentum, and $\mathrm{F}$ is a weight update function, which indicates how weights are changed during the learning process. There is no single best weight update function, which can be applied for all non-linear optimizations. One needs to choose a weight update function based on the characteristics of the problem and the data set of interest. Various types of algorithms have been found to be effective for most practical purposes. However, two different weight update functions of Levenberg-Marquardt (LM) algorithm and Scaled Conjugate Gradient (SCG) are used in this study.

The Levenberg-Marquardt algorithm appears to be the fastest method for training moderate-sized feedforward neural networks. The LevenbergMarquardt algorithm was designed to approach second-order training speed without having to compute the Hessian matrix. The conjugate gradient algorithms require a line search at each iteration. This line search is computationally expensive, because it requires that the network response to all training inputs be computed several times for each search. The scaled conjugate gradient algorithm (SCG) was designed to avoid the time-consuming line search. The details about these algorithms can be found elsewhere ${ }^{30}$.

\section{COMPUTATIONAL METHOD}

\section{Data set}

Fifty substituted phenol derivatives that have been used as study materials are given in Table 1 along with their observed toxicity $(\mathrm{pC})$ over tetrahymena pyriformis. The toxicity values have been taken from the literature [29]. The toxicity values have been used as the corresponding $\mathrm{pC}$ values. For regression analysis (PCR and PLS), this set was divided randomly into two sets including training (calibration) set ( 30 compounds) and test set ( 20 compounds) and for ANN analysis, this set was divided into three sets including training (calibration) set (30 compounds), test set (10 compounds) and validation set (10 compounds).

Table 1 Observed and calculated values of toxicity using PCR, PLS and ANNs methods- Training (calibration), Validation and Test sets (Note: for PCR and PLS models, both of validation and test sets are placed as test set

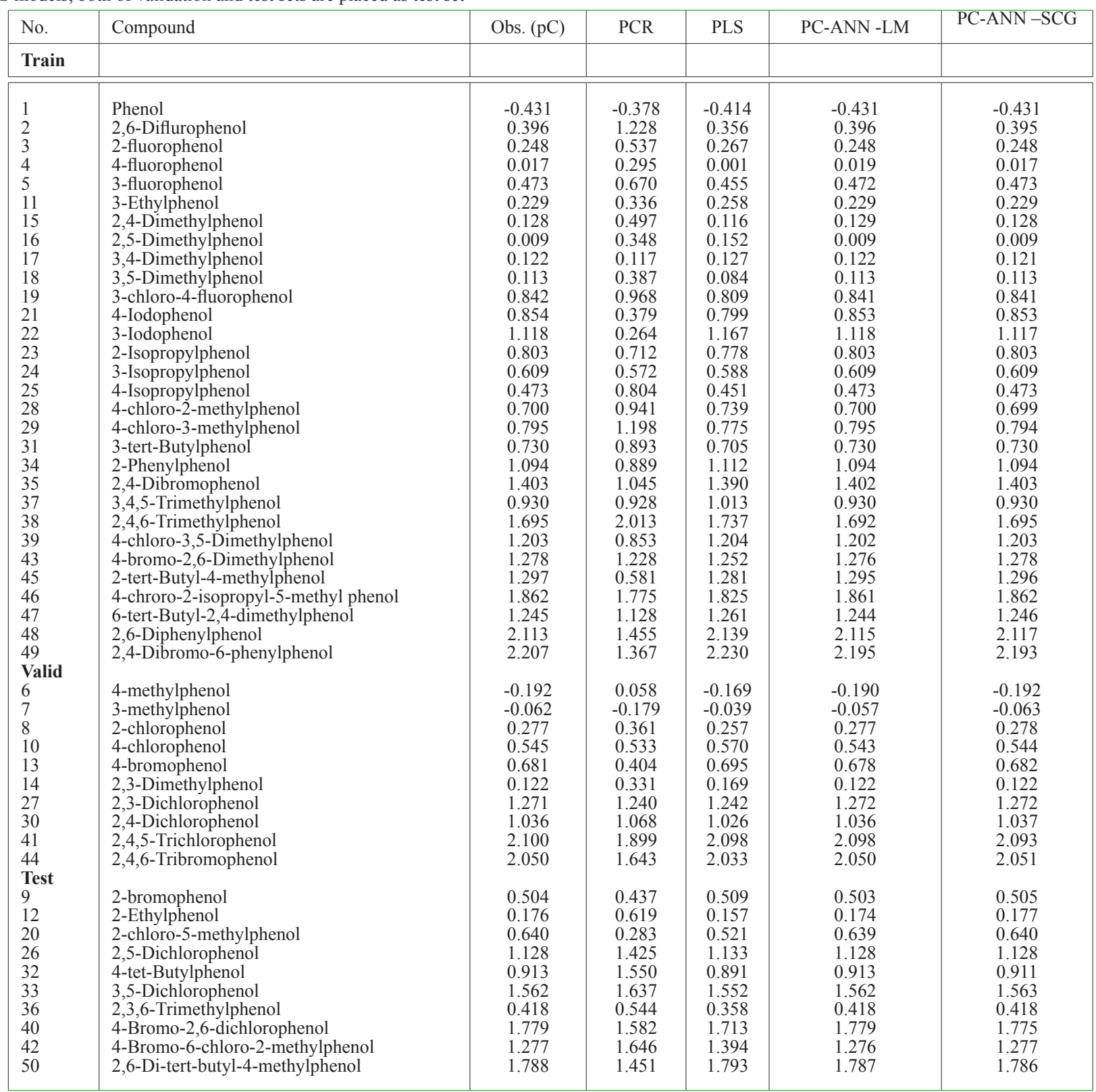




\section{Multivariate image analysis descriptors}

The structures of each compound were systematically drawn in the ChemSketch module of ACDLabs program ${ }^{31}$ and saved in paint brush as bmp files, and then converted to bitmaps in a $210 \times 150$ pixels workspace, with a resolution of $72 \times 72$ dots per inch. All the molecular structures were fixed by a common point among them in a given coordinate $(100,50)$, since the shapes should be superimposed afterwards, as a $2 \mathrm{D}$ alignment to allow maximum similarity, as illustrated in Figure 1. Each 2D image was read and converted into binary data (a double array in Matlab), and the three-way array, the predictors block, was built by grouping the 50 treated images, giving a 50 $\times 210 \times 150$ array. The $3 \mathrm{D}$ array was unfolded to a two-way array, the $\mathrm{X}$-matrix, $(50 \times 31500)$. However, to minimize the memory used, columns with zero variance were removed, reducing the $\mathrm{X}$ dimension to $50 \times 968$.

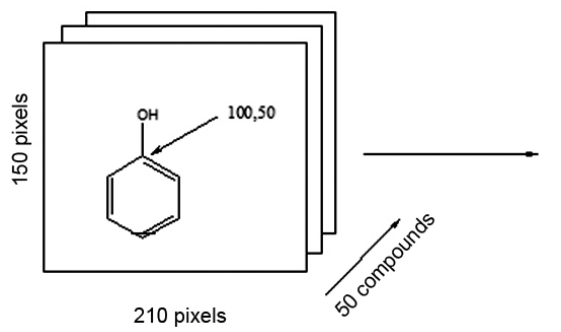

31500 pixels

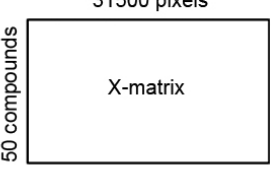

Figure 1 Superposition of the 50 chemical structures (2D images) and unfolding step to give the X-matrix. The arrow in structure indicates the coordinate of a pixel in common among the whole series of compounds, used in the $2 \mathrm{D}$ alignment step.

\section{Partial Least Square analysis}

The key before PLS analysis is the proper reorganization of the original 3-way array. In the previous step, unfolding is done so that pixels become a single row and, thus, an image that is originally I by J pixels for K compounds

Table 2 Results of CR-PCR in the presence of different entered PCs

\begin{tabular}{|c|c|c|c|c|c|}
\hline PC entered & SE & $\mathbf{R}^{2}$ & $\mathbf{R}^{2}$ (Adj) & PRESS & $\mathbf{R}^{2}$ (Pred) \\
\hline $\mathrm{C} 2$ & 0.584 & $24.3 \%$ & $22.7 \%$ & 17.83 & $17.74 \%$ \\
\hline $\mathrm{C} 2+\mathrm{C} 24$ & 0.586 & $25.6 \%$ & $22.4 \%$ & 18.11 & $16.45 \%$ \\
\hline $\mathrm{C} 2+\mathrm{C} 24+\mathrm{C} 5$ & 0.568 & $31.4 \%$ & $26.9 \%$ & 17.52 & $19.19 \%$ \\
\hline $\mathrm{C} 2+\mathrm{C} 24+\mathrm{C} 5+\mathrm{C} 18$ & 0.549 & $37.2 \%$ & $31.7 \%$ & 16.56 & $23.63 \%$ \\
\hline $\mathrm{C} 2+\mathrm{C} 24+\mathrm{C} 5+\mathrm{C} 18+\mathrm{C} 15$ & 0.534 & $42.1 \%$ & $35.6 \%$ & 15.53 & $28.38 \%$ \\
\hline $\mathrm{C} 2+\mathrm{C} 24+\mathrm{C} 5+\mathrm{C} 18+\mathrm{C} 15+\mathrm{C} 12$ & 0.519 & $46.5 \%$ & $39.0 \%$ & 15.12 & $30.24 \%$ \\
\hline $\mathrm{C} 2+\mathrm{C} 24+\mathrm{C} 5+\mathrm{C} 18+\mathrm{C} 15+\mathrm{C} 12+\mathrm{C} 6$ & 0.506 & $50.2 \%$ & $42.0 \%$ & 14.67 & $32.33 \%$ \\
\hline $\mathrm{C} 2+\mathrm{C} 24+\mathrm{C} 5+\mathrm{C} 18+\mathrm{C} 15+\mathrm{C} 12+\mathrm{C} 6+\mathrm{C} 14$ & 0.493 & $53.9 \%$ & $44.9 \%$ & 13.95 & $35.66 \%$ \\
\hline $\mathrm{C} 2+\mathrm{C} 24+\mathrm{C} 5+\mathrm{C} 18+\mathrm{C} 15+\mathrm{C} 12+\mathrm{C} 6+\mathrm{C} 14+\mathrm{C} 17$ & 0.48 & $57.4 \%$ & $47.8 \%$ & 12.87 & $40.61 \%$ \\
\hline $\mathrm{C} 2+\mathrm{C} 24+\mathrm{C} 5+\mathrm{C} 18+\mathrm{C} 15+\mathrm{C} 12+\mathrm{C} 6+\mathrm{C} 14+\mathrm{C} 17+\mathrm{C} 16$ & 0.469 & $60.4 \%$ & $50.2 \%$ & 12.82 & $40.88 \%$ \\
\hline $\mathrm{C} 2+\mathrm{C} 24+\mathrm{C} 5+\mathrm{C} 18+\mathrm{C} 15+\mathrm{C} 12+\mathrm{C} 6+\mathrm{C} 14+\mathrm{C} 17+\mathrm{C} 16+\mathrm{C} 9$ & 0.459 & $63.0 \%$ & $52.3 \%$ & 12.96 & $40.23 \%$ \\
\hline $\mathrm{C} 2+\mathrm{C} 24+\mathrm{C} 5+\mathrm{C} 18+\mathrm{C} 15+\mathrm{C} 12+\mathrm{C} 6+\mathrm{C} 14+\mathrm{C} 17+\mathrm{C} 16+\mathrm{C} 9+\mathrm{C} 11$ & 0.45 & $65.3 \%$ & $54.1 \%$ & 13.00 & $40.02 \%$ \\
\hline $\mathrm{C} 2+\mathrm{C} 24+\mathrm{C} 5+\mathrm{C} 18+\mathrm{C} 15+\mathrm{C} 12+\mathrm{C} 6+\mathrm{C} 14+\mathrm{C} 17+\mathrm{C} 16+\mathrm{C} 9+\mathrm{C} 11+\mathrm{C} 40$ & 0.44 & $67.6 \%$ & $56.0 \%$ & 12.73 & $41.26 \%$ \\
\hline $\mathrm{C} 2+\mathrm{C} 24+\mathrm{C} 5+\mathrm{C} 18+\mathrm{C} 15+\mathrm{C} 12+\mathrm{C} 6+\mathrm{C} 14+\mathrm{C} 17+\mathrm{C} 16+\mathrm{C} 9+\mathrm{C} 11+\mathrm{C} 40+\mathrm{C} 41$ & 0.433 & $69.7 \%$ & $57.5 \%$ & 12.78 & $41.04 \%$ \\
\hline $\mathrm{C} 2+\mathrm{C} 24+\mathrm{C} 5+\mathrm{C} 18+\mathrm{C} 15+\mathrm{C} 12+\mathrm{C} 6+\mathrm{C} 14+\mathrm{C} 17+\mathrm{C} 16+\mathrm{C} 9+\mathrm{C} 11+\mathrm{C} 40+\mathrm{C} 41+\mathrm{C} 47$ & 0.426 & $71.5 \%$ & $58.9 \%$ & 12.38 & $42.91 \%$ \\
\hline
\end{tabular}

The data set was divided into a representative training set including 30 compounds and test set including 20 compounds.

\section{CR-PC-ANN modeling}

Two different ANN models including Levenberg-Marquardt and Scaled Conjugate Gradient (LM and SCG) algorithms were employed in this work. Our networks had an input layer, a hidden layer and an output layer. The input vectors were the set of PCs which selected by correlation ranking. Number of nodes in the input layer was depended on the number of PCs introduced in the network. The number of nodes in the hidden layer was optimized through learning procedure. There was only one node in the output layer. The whole data set was divided into a representative training set including 30 compounds, test set including 10 compounds and validation set including 10 compounds. The training and test data sets were used to optimize the network performance.

\section{RESULTS AND DISCUSSION}

3D QSTR analysis requires knowledge about main conformation and is reshaped to form a two way array that is I $\times \mathrm{J}$ by k. an $\mathbf{X}$-matrix is then built, where each row contains the variables (the pixels) describing each molecule, and is subsequently decomposed into a score vector $\mathbf{s}_{1}$ and a weight vector $\mathbf{w}_{1}$. The score vector is determined to have the property of maximum covariance with the dependent variable $\mathbf{y}$. The score vectors then replace the original variables as regressors. In the present analysis, modeling by PLS method was performed using MINITAB $15^{32}$. For regression analysis, data set was separated into two groups: training and test sets (Table 1). The number of significant factors for the PLS algorithm was determined using the leave-one-out cross-validation method. The predicted values of left-out samples were then compared to the observed values using prediction error sum of squares (PRESS). The PRESS obtained in the cross-validation was calculated each time that a new principal component (PC) was added to the model. The optimum number of PLS factors is the one that minimizes PRESS. The best PLS model contained 4 factors.

\section{CR-PCR modeling}

In this section, the correlation coefficient between each of the PCs and toxicity is included. The correlation coefficient is a part of variance in the dependent variables (toxicity) which is explained by a PC. PCs with higher correlation coefficient have greater information about the variation in the toxicity. The decreasing order of correlation coefficients of the PCs with toxicity is: $\mathrm{PC} 2>\mathrm{PC} 24>\mathrm{PC} 5>\mathrm{PC} 18>\mathrm{PC} 15>\mathrm{PC} 12>\mathrm{PC} 6>\ldots$ This trend shows that $\mathrm{PC} 1$ and $\mathrm{PC} 3 \ldots$ which have high information content about the descriptors do not have useful information about toxicity. In the CR-PCR equation, the factor with highest correlation coefficient with the toxicity is considered as the most significant one and, subsequently, the factors are introduced into the calibration model until no further improvement of the calibration model is obtained. The statistical results of CR-PCR in the presence of different entered PCs are shown in Table 2. Final selected CR-PCR equation is as follow:

Toxicity $=0.851( \pm 0.060)+0.0253( \pm 0.005) \mathrm{PC} 2-0.0252( \pm 0.021)$ PC24 - 0.0223( \pm 0.008$)$ PC5 $+0.0436( \pm 0.016)$ PC18 $-0.0361( \pm 0.015) \mathrm{PC} 15$ - $0.0314( \pm 0.014)$ PC12 - $0.0204( \pm 0.009)$ PC6 $+0.0302( \pm 0.014)$ PC14 + $0.0323( \pm 0.016) \mathrm{PC} 17+0.0292( \pm 0.015) \mathrm{PC} 16-0.0211( \pm 0.012) \mathrm{PC} 9-$ $0.0220( \pm 0.013)$ PC1 $-0.0522( \pm 0.031)$ PC40 - $0.0508( \pm 0.032)$ PC41 - 0.0606 $( \pm 0.041) \mathrm{PC} 47$

(2)

alignment of molecules in order to generate descriptors suitable to represent interactions such as steric, electrostatic and hydrogen bonds. This treatment is often exhaustive or even impracticable for molecules with a large size. At this point, utilization of $2 \mathrm{D}$ images to generate descriptors avoids these complications, since no specific tool or high computational investments are needed. 2D image of a molecule does not represent particular effects, for instance steric interactions or any physicochemical parameter, but it is a general manner to represent any property, since small difference in drawing a structure can cause significant changes in the predicted property, i.e. each pixel location acts as a code (the descriptors are binary).

The main aim of the present work was to develop MIA-QSTR models to predict toxicity of phenol derivatives against Tetrahymena pyriformis. Table 1 lists the compounds used in this study and their corresponding experimental toxicity. In order to find a correlation between MIA descriptors and toxicity, MIA-QSTR models were built and the calibration step was carried out through PLS, CR-PCR and CR-PC-ANN methods.

To obtain accurate models, relevant information was extracted from the 
X-matrix by selecting suitable regressors. The MIA-QSTR method, when coupled to the well-known Partial Least Square (PLS) regression methods, has presented prediction results comparable to $3 \mathrm{D}$ methodologies. The $2 \mathrm{D}$ alignment is simpler than a 3D one. The estimated toxicity using PLS method is shown in Table 1. Statistical parameters including squared correlation coefficient $\left(\mathrm{R}^{2}\right)$, standard error (SE) and relative errors (RE) for this method are indicated in Table 3 for training (calibration) and test sets, respectively.

Table 3 The statistical parameters for ANNs, PLS and PCR models.

\begin{tabular}{|c|c|c|c|c|c|c|c|c|c|}
\hline \multirow{2}{*}{$\begin{array}{c}\text { Set } \\
\text { Method }\end{array}$} & \multicolumn{2}{|c|}{$\begin{array}{c}\text { Training } \\
\text { (Calibration }\end{array}$} & \multirow[b]{2}{*}{$\mathrm{REC} \%$} & \multicolumn{2}{|r|}{ Test } & \multicolumn{3}{|c|}{ Validation } & \multirow[b]{2}{*}{ REV\% } \\
\hline & $\mathrm{R}^{2}$ & SE & & $\mathrm{R}^{2}$ & $\mathrm{SE}$ & RET\% & $\mathrm{R}^{2}$ & $\mathrm{SE}$ & \\
\hline PCR & 0.621 & 0.401 & 47.12 & 0.837 & 0.291 & 34.19 & - & - & \\
\hline PLS & 0.996 & 0.042 & 4.93 & 0.996 & 0.048 & 5.64 & - & - & \\
\hline PC-ANN-LM & 0.960 & 0.128 & 15.04 & 0.651 & 0.364 & 42.77 & 0.876 & 0.306 & 35.95 \\
\hline PC-ANN-SCG & 0.999 & 0.002 & 0.23 & 0.999 & 0.001 & 0.12 & 0.999 & 0.002 & 0.23 \\
\hline Previous work-AM1 ${ }^{\mathrm{a}}$ & 0.881 & 0.241 & & & & & & & \\
\hline Previous work-PM3 a & 0.853 & 0.270 & & & & & & & \\
\hline Previous work-PM5 a & 0.857 & 0.267 & & & & & & & \\
\hline Previous work-DFT ${ }^{\mathrm{a}}$ & 0.910 & 0.210 & & & & & & & \\
\hline
\end{tabular}

a Taken from reference 29.

In the PCA-ranking method, the criterion for the selection of the space of the PCs containing the important response matrix is the correlation of the PCs with toxicity. Therefore, we have inspected the scores in the space of the correlated PCs for choosing the response matrix with highest variances in this space. The selected descriptors were regressed against the toxicity through PCR. In due course, linear equation (2) has been developed whose specifications are given in Table 3. Calculated toxicities using CR-PCR model are shown in Table 1. Statistical parameters including squared correlation coefficient $\left(\mathrm{R}^{2}\right)$ and standard error (SE) for this model are indicated in Table 3 for training and test sets, respectively. This model has a prediction power with $\mathrm{R}^{2}=0.837$ and $\mathrm{SE}=0.291$ for test set.

To increase the prediction ability of the obtained model between the PCs and toxicity, a non-linear modeling method was employed in this study. Typically, superior models can be found using ANNs because they implement non-linear relationships and because they have more adjustable parameters than the linear models. Therefore, in this study we suggested the use of ANNs as the non-linear model. In the same manner as PCR analysis, correlation ranking was used to select the most relevant set of PCs as input of ANNs. It is a common practice to optimize the parameters of number of nodes in the hidden layer, learning rate and momentum in developing a reliable network. However, as it can be seen from Eq. (1), there is a term called weight update function, which indicates the way that weights are changed during the learning process. In this paper, the role of weight update function in artificial neural networks was investigated. The calculated values of toxicity for training, test and validation sets using two different LM and SCG -ANNs are shown in Table 1. The statistical parameters of $\mathrm{R}^{2}$ and standard error (SE) for these two algorithms are given in Table 3 for training, test and validation sets, respectively. It can be seen from Table 3 that there is an excellent correlation between estimated toxicity and the experimental data. Based on the data given in Table 3, a comparison between the results obtained by the ANNs, PLS and PCR methods clearly indicates the superiority of ANNs and PLS over that of the PCR model.

Comparison of the results for different ANN algorithms indicates that the scaled conjugate gradient algorithm (SCG) has the best performance on this problem, although Levenberg Marquardt algorithm performs well, too. The conjugate gradient algorithms, in particular SCG, seem to perform well over a wide variety of problems, particularly for networks with a large number of weights. The SCG algorithm is almost as fast as the LM algorithm on function approximation problems (faster for large networks). The conjugate gradient algorithms have relatively modest memory requirements. Figures 2 (a-c) demonstrates the plot of the predicted versus the experimental values of the toxicity using PLS and two different ANN algorithms. Correlation coefficients of these plots indicate the reliability of the models.

Comparison of the obtained results in this work with the results of a previous study [29] where 3D QSTR was used for generation of descriptors indicates the superiority of our results. For comparing, statistical parameters of this study are also shown in Table 3.

In summary, the obtained results in our study indicate that the use of molecular modeling may be useful for the understanding of the toxicological behavior of molecules. MIA descriptors are capable to recognize the physicochemical information and may be useful to predict toxicity.

$\mathbf{a}$

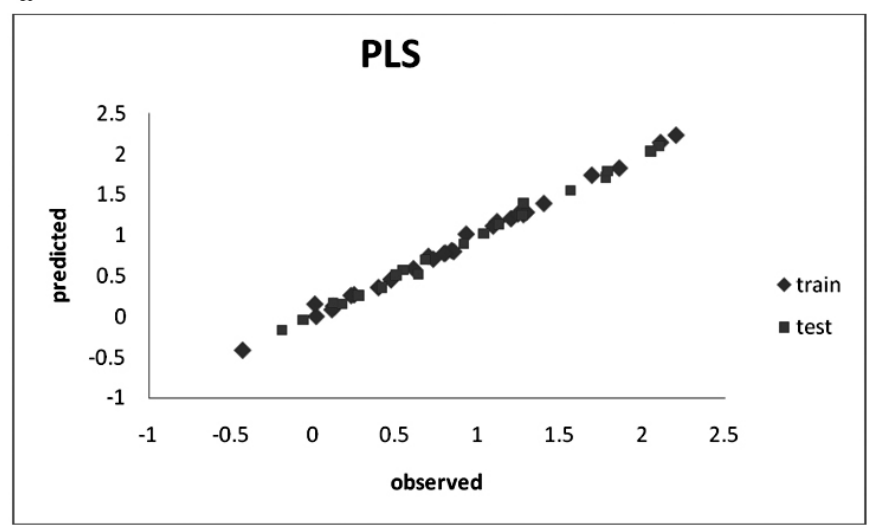

b

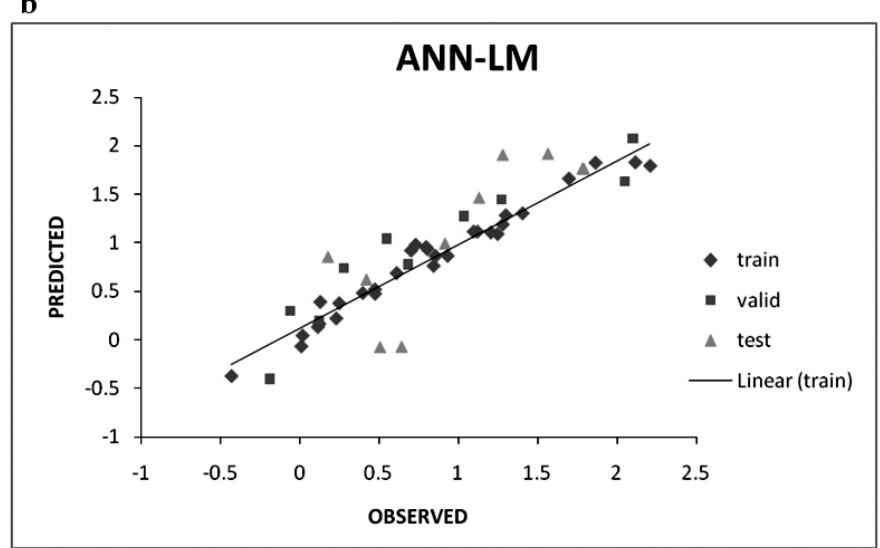


c

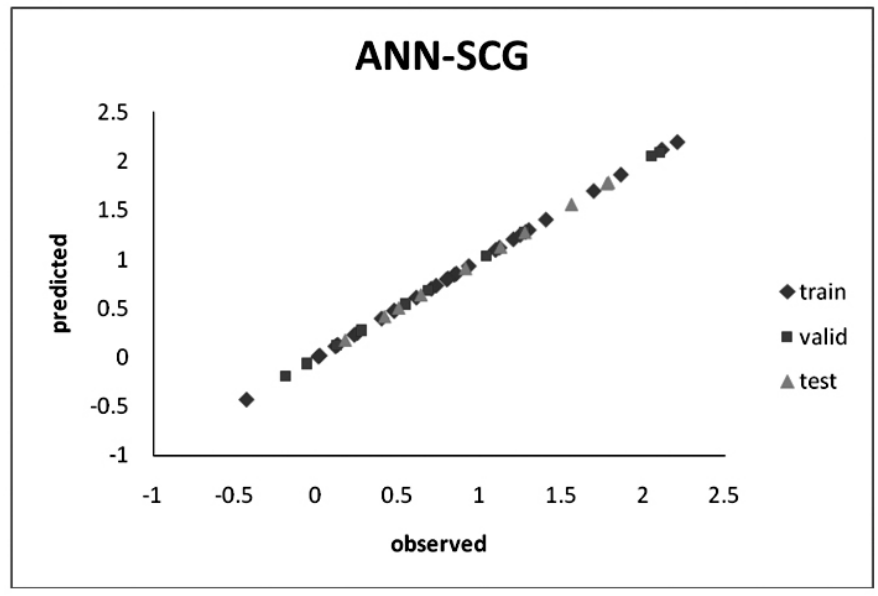

Figure 2 Plot of experimental toxicity of phenol derivatives against the calculated values using a) PLS, b) ANN-LM and c) ANN-SCG models

\section{CONCLUSION}

A quantitative-toxicity relationship analysis has been conducted on the toxicities of 50 different phenol derivatives by using partial least square regression, principal component regression and principal component-artificial neural network modeling methods with application of correlation ranking. MIA descriptors and the methodology presented here can clearly be applied for the estimation of toxicity of phenol derivatives with high predictive power. The study is helpful in the determination of the effect of any particular phenol derivative of this series over Tetrahymena pyriformis. Superiority of nonlinear models (ANNs) over the linear model (PCR) revealed that the toxicity of phenol derivatives has non-linear characteristics when MIA descriptors are used.

\section{REFERENCES}

1. T. M. Lin, S. S. Lee, C. S. Lai, S. D. Lin, J. Inter. Soc. Burn Injuries. 32, 517, (2006)

2. M. A. Warner, J. V. Harper, Anesthesiology 62, 366, (1985)

3. C. Hansch, S. C. Mc Karns, C. J. Smith, D. J. Doolittle, Chemico-Bio.
Interact. 127, 61, (2000)

4. H. Kubinyi, Drug. Develop. Theory 2, 457 (1997)

5. D. Hadjipavlou-Litina, Med. Res. Rev. 18, 91, (1998)

6. P. Gramatica, E. Papa, QSAR Comb. Sci. 22, 347, (2003)

7. D. C. Montogomery, E. A. Peck, Introduction to linear analysis, Wiley, New York, 1982.

8. I. T. Jolliffie, Principal component analysis, Springer, New York, 1986.

9. J. H. Kalivas, P. M. Lang, Mathematical analysis of spectral orthogonality, Marcel Dekker, New York, 1994.

10. G. Puchwein, Anal. Chem. 60, 569, (1988)

11. Y.L. Xie, J. H. Kalivas, Anal. Chim. Acta. 348, 19, (1997)

12. U. Depczynski, V. J. Frost, K. Molt, Anal. Chim. Acta. 420, 217, (2000)

13. A. S. Barros, D. N. Rutledge, chemomet. Intell. Lab. Syst. 40, 65, (1998)

14. J. M. Sutter, J. H. Kalivas, P. M. Lang, J. Chemometr. 40, 65, (1992)

15. J. Sun, J. Chemometr. 9, 21, (1995)

16. K. Petritis, L. J. Kangas, P. L. Ferguson, G. Anderson, L. Pasa-Tolic, M.s. Lipton, K. J. Auberry, E.F. Strittmatter, Y. Shen, R. Zhao, R. D. Smith, Anal. Chem. 75, 1039, (2003)

17. L. Douali, D.Villemin and D. Cherqaoui, J. Chem. Inf. Comput. Sci. 43, 1200, (2003)

18. J. Polanski, J. Gasteiger, M. Wagener, J.Sadowski, Quant. Struct. Act. Relant. 17, 27, (1998)

19. B. Hemmateenejad, M. A. Safapour, F. Taghavi, J. Mol. Sruct (Theochem). 635, 183, (2003)

20. M. Samsipour, B. hemmateenejad, M. Akhond, Anal. Chim. Acta. 461, $147,(2002)$

21. D. T. Manallack, B. G. Tehan, E. Gancia, B. D. Hudson, M. G. Ford, D. J. Livingstone, D. C. Whitley, W. R. Pitt, J. Chem. Inf. Comput. Sci. 43, 674, (2003)

22. Schneider, P. Wrede, Prog. Biophys. Mol. Biol.70, 175, (1998)

23. P. J. Gemperline, J. R. Long, G. Gregoriou, Anal. Chem. 63, 2313, (1991)

24. R. Vendrame, R. S. Braga, Y. Takahata, D. S. Galvao, J. Chem. Inf. Comput. Sci. 39, 1094, (1999)

25. P. Geladi, S. Wold, K. Esbensen, Anal. Chim. Acta. 191, 473, (1986)

26. P. Geladi, K. Esbensen, J. Chemometr. 5, 97, (1991)

27. P. Geladi, H. Grahn, Multivariate image analysis, Wiley, Chichester, UK, 1996.

28. K. H. Esbensen, K. H. Hjelmen, K. Kvaal, J. Chemometr. 10, 569, (1996)

29. F.A. Pasha, H.K. Srivastava, P.P. Singh, J. BMC. 13, 6823, (2005)

30. MATLAB version 7.1 Mathworks Inc., (2005), www.Mathworks.com

31. $\mathrm{ACD} /$ ChemSketch version 12 , Advanced chemistry development Inc., Toronto, Ont., Canada, 1997-1998.

32. MINITAB, Version 15.1, (2008), www.minitab.com 